\title{
Krankenhausfinanzierung und -vergütung als politisches Handlungsfeld
}

Nils C. Bandelow, Johanna Hornung und Lina Y. Iskandar

16.1 Ausgangslage -300

16.2 Krankenhausfinanzierung im kooperativen Föderalismus - 302

16.3 Interessengruppen und Selbstverwaltung - 304

16.4 Krankenhauspolitik in der Parteien-Arena - 306

16.5 Ausblick - 309

Literatur - 311 


\section{- Zusammenfassung}

Krankenhauspolitik ist von Zielkonflikten zwischen verschiedenen staatlichen Ebenen, Parteien, Akteuren der Selbstverwaltung, Krankenhausträgern, Krankenhausbeschäftigten und Patienteninteressen geprägt. Diese sind auf unterschiedlichen Ebenen in die aktuellen Diskussionen um Fallpauschalen im Besonderen und Krankenhausfinanzierung und -vergütung im Allgemeinen eingebunden. Der Beitrag beleuchtet das für die Krankenhauspolitik relevante institutionelle Gefüge des kooperativen Föderalismus und die darin eingebetteten Akteurskonstellationen im Hinblick auf ihre Interessen und die Möglichkeiten, diese Interessen durchzusetzen. Wie sind die politischen Entwicklungen der letzten zehn Jahre und die aktuelle Situation aus politikwissenschaftlicher Perspektive $z u$ bewerten? Inwiefern kooperieren oder blockieren sich die beteiligten Akteure und politischen Ebenen? Welche Reformalternativen versprechen effiziente Steuerung und Konfliktminimierung unter Berücksichtigung aller Interessen? Diese Bestandsaufnahme resultiert in einem politikwissenschaftlichen Ausblick mit Empfehlungen und Forschungsanreizen zur zukünftigen Finanzierungs- und Vergütungsgestaltung in der Krankenhauspolitik.

Hospital policy in Germany is characterised by different conflicting objectives between various levels of governance, political parties, self-governance, hospital operators, hospital employees and patients' interests. These are involved at various levels in the current discussions on DRGs in particular and hospital financing and remuneration in general. This article examines the institutional structure of cooperative federalism relevant to hospital policy and the constellations of stakeholders embedded in it with regard to their interests and the possibilities of enforcing these interests. How can the political developments of the last ten years and the current situation be assessed from a political science perspective? To what extent do the actors and political levels involved cooperate or block each other? Which reform alternatives promise efficient control and conflict minimisation, considering all interests?
This review results in an outlook with recommendations and research incentives for financing and remuneration in hospital policy in the future.

\subsection{Ausgangslage}

Das Fallpauschalensystem war aus politischer Sicht das Ergebnis von langjähriger Vorbereitung unter unterschiedlichen Regierungskoalitionen, der formalen Verabschiedung im Jahr 2000 mit dem GKV-Gesundheitsreformgesetz bis zum sich einstellenden Regelbetrieb im Jahr 2010 (Simon 2016). Eingebettet in ein größeres Programm kann es auch als politisches Instrument einer programmatischen Gruppe gesehen werden, die im Gesundheitswesen die Vision von Wettbewerb in einer solidarischen Rahmenordnung verfolgte (Hornung und Bandelow 2018). Obwohl das Fallpauschalensystem kontinuierlich angepasst wird - in jüngster Zeit etwa durch die Exklusion der Pflegekosten - ist ein radikaler Wandel des Vergütungssystems nur schwer möglich, wie die lange Vorbereitungszeit zur Einführung der Fallpauschalen beweist. Dies liegt unter anderem an der Komplexität von Akteurskonstellationen: In der deutschen Gesundheitspolitik konkurrieren Krankenhausträger, Beschäftigte, Kostenträger, Patienten und Akteure aus Kommunal-, Landes- und Bundespolitik mit teils stark divergierenden Interessen um Einfluss und Entscheidungsmacht. Dabei ist die Krankenhausfinanzierung und -vergütung ein zentraler Streitpunkt in unterschiedlichen Konfliktlinien, innerhalb derer sich die von den Akteuren verfolgten Ziele an zum Teil gegensätzlichen Dimensionen orientieren (Bandelow et al. 2009).

Aufgrund des für Deutschland charakteristischen kooperativen Föderalismus ist die Kompetenzverteilung zwischen Bund, Ländern und Kommunen erstens Gegenstand einer föderalen Konfliktlinie. Je nach Ebene gibt es gesonderte Kompetenzen in der Krankenhausfinanzierung und -vergütung. Auf der Bundesebene werden vor allem die all- 
gemeinen Rahmenbedingungen festgesetzt, etwa der dualen Finanzierung von Betriebskosten (durch Kostenträger) und Investitionskosten (durch Bundesländer). Während die Bundesebene bspw. über den Kommunalinvestitionsförderungsfonds oder bundesweit geltende Krankenhaus-Fallpauschalenverordnungen Einfluss auf Finanz- und Vergütungsstrukturen ausübt, sind die Bundesländer - hier vor allem die Krankenhausplanungsausschüsse der Landesparlamente - durch den Sicherstellungsauftrag mit Krankenhausplanung und Investitionsprogrammen betraut. Bund, Länder, Kommunen und weitere Gebietseinheiten bilden gleichzeitig auch die öffentlichen Trägerschaften $\mathrm{ab}$, deren Interessen sich untereinander und auch im Vergleich zu den anderen Trägern (freigemeinnützig und privat) unterscheiden. Die Kommunen sind darüber hinaus entsprechend den landesspezifischen Krankenhausgesetzen an der Investitionsfinanzierung von Krankenhäusern beteiligt. Der Förderanteil ist in Niedersachsen und Nordrhein-Westfalen etwa auf $40 \%$ festgelegt - diesen Anteil tragen die Kommunen an der Finanzierung der Investitionskosten in Krankenhäusern. Aufgrund der teils stark wachsenden Haushaltsdefizite beklagen die Kommunen jedoch regelmäßig die Mitwirkung an dieser Finanzierung.

In diese institutionellen Strukturen eingebettet sind aber auch andere Interessengruppen und Selbstverwaltungsakteure; im Krankenhausbereich sind es insbesondere die Deutsche Krankenhausgesellschaft, Spitzenverbände von gesetzlichen und privaten Krankenkassen sowie Berufsverbände, vor allem Ärzteund in Zukunft auch Pflegekammern (Blum 2018), die diese Entscheidungsarenen unterschiedlich erfolgreich bespielen.

Zweitens lässt sich eine ökonomische Konfliktlinie beobachten, die hauptsächlich entlang der Interessen von Kostenträgern und Leistungsempfängern verläuft. Kostenträger sind in der Krankenhauspolitik vor allem die gesetzlichen Krankenkassen, die private Krankenversicherung und staatliche Akteure (vor allem die Bundesländer), die eine effiziente Ausgabensteuerung und -senkung befürworten. Die
Beschäftigten im Krankenhaussektor streben nach einem angemessenen Verdienst zur Bestreitung ihres Lebensunterhalts, während $\mathrm{Pa}$ tienten eine hohe Qualität und gute Erreichbarkeit der Versorgung priorisieren. Durch das Sachleistungsprinzip sind letztere zudem am wenigsten mit Finanzierungs- und Vergütungsstrukturen vertraut. Effizienzgewinne sind im Krankenhaus durch das Uno-ActuPrinzip kaum möglich (Matusiewicz 2019): Die Tatsache, dass gleichzeitig personenbezogene Dienstleistungen erbracht und empfangen werden, beschränkt die Möglichkeit, Personal durch technischen Fortschritt einzusparen. Will man Krankenhauspersonal am Effizienzgewinn anderer Sektoren beteiligen, ergibt sich daraus ein kontinuierlicher Kostendruck, d.h. der Anteil der Krankenhausausgaben am Bruttoinlandsprodukt und an den gesamten Gesundheitsleistungen würde ohne politische Maßnahmen steigen. Daraus ergibt sich die besondere Herausforderung, Anreize für die bestmögliche Versorgung zu setzen und gleichzeitig eine angemessene Vergütung für die Beschäftigten zu sichern, ohne übermäßige Kostensteigerungen zuzulassen.

Drittens besteht vor allem in parteipolitischen Auseinandersetzungen ein Diskurs, der sich auf eine normative Konfliktlinie zurückführen lässt. Historisch beschreibt diese einen Konflikt zwischen Anhängern der Solidarität, die sich häufig den linken, sozialdemokratischen und teilweise grünen Parteien zuordnen, und Befürwortern von Eigenverantwortung, etwa Christdemokraten und Liberale, als Priorität für die Organisation des deutschen Gesundheitswesens. Dieser normative Konflikt prägte vor allem in den 1970er und 1980er Jahren gesundheitspolitische Bündnisse und schlug sich auch noch in der Diskussion um Gesundheitsprämie und Bürgerversicherung nieder, die schließlich durch die Einführung des Gesundheitsfonds 2007 aufgelöst wurde. Folglich tun sich seither aufkommende, erst in den letzten Jahren zunehmend erfolgreiche oder fusionierte Parteien mit einer klaren Zuordnung schwer, da sich dieser Gegensatz primär zwischen den Linien der SPD und Lin- 
ken (sowie teilweise Bündnis 90/Die Grünen) auf der einen Seite und den Positionen der CDU/CSU und FDP auf der anderen Seite vollzog. Die AfD ist noch auf der Suche nach einem kohärenten gesundheitspolitischen Profil (Paquet 2019). Aktuell bezieht sich der Konflikt in einer abgeschwächten Form auf die Frage, wer einen berechtigten Zugang zu gesundheitsbezogenen Leistungen hat, welchen Umfang diese haben und wie deren Finanzierung gestaltet wird, etwa durch private Zuzahlungen, Beitragsgelder (Stichwort GKV-Leistungskatalog) oder auf Bundesebene entschiedene Steuerzuschüsse.

Quer zu diesen Konfliktlinien wird vor allem in der letzten Dekade zunehmend ein Qualitätsdiskurs geführt, der alle Bereiche schneidet und die Krankenhauspolitik häufig überlagert (Bandelow et al. 2009). Pflegepersonaluntergrenzen und qualitativ hochwertige Behandlung infolge richtiger Diagnosen sind nur ausgewählte Beispiele für Qualitätsthemen, die unmittelbar mit Finanzierung und Vergütung in Zusammenhang stehen. Das Qualitätskonzept steht jedoch - anders als bspw. die Ziele Finanzierbarkeit, Solidarität und (wirtschaftliches) Wachstum - vor der Herausforderung einer einheitlichen Definition, Operationalisierbarkeit und Erreichbarkeit. Aus normativer Perspektive ist es zudem öffentlich nicht vertretbar, abzulehnen, dass dieses Qualitätskonzept prioritär ist. Lediglich über die Umsetzung lässt sich streiten.

Veränderungen innerhalb des Fallpauschalensystems sind nur inkrementell und langfristig möglich sowie ggf. als Teilinstrument in einem planbaren politischen Programm umsetzbar, das in der aktuellen Legislaturperiode jedoch nicht erkennbar ist (Heyder und Malzahn 2018). Vor diesem Hintergrund analysiert dieser Beitrag die politischen Entwicklungen in der Krankenhausfinanzierung und -vergütung und bewertet die aktuelle Situation aus politikwissenschaftlicher Perspektive. Inwiefern die genannten politischen Ebenen und beteiligten Akteure in diesem Kontext als Kooperations- oder Konfliktpartner agieren, wird in den folgenden Kapiteln systema- tisch anhand der beschriebenen Konfliktlinien nachgezeichnet. Die Analysen münden in einem Ausblick für die Bedeutung und Ausgestaltung zukünftiger Krankenhauspolitik in politischen Prozessen. Dieser beinhaltet eine Bewertung von Reformalternativen, die eine effiziente Steuerung und Konfliktminimierung versprechen.

\subsection{Krankenhausfinanzierung im kooperativen Föderalismus}

Der kooperative Föderalismus in Deutschland gibt den Bundesländern eine besondere Rolle in der Krankenhauspolitik. Aufgrund der konkurrierenden Gesetzgebung liegt die wirtschaftliche Sicherung der Krankenhäuser und die Regelung der Krankenhauspflegesätze im Kompetenzbereich der Bundesländer, sofern es keine bundesgesetzlichen Bestimmungen gibt und sofern der Bund nicht durch Gesetzgebungstätigkeit in diesem Bereich für die Herstellung gleichwertiger Lebensverhältnisse oder Wahrung einer Rechts- oder Wirtschaftseinheit im gesamtstaatlichen Interesse sorgen muss (Art. 72 Abs. 1-2 GG; Art 74 Abs. 19a GG). Für die Krankenhauspolitik bedeutet diese Einschränkung konkret, dass bspw. finanzielle Schwierigkeiten von Krankenhäusern in einem Bundesland dazu führen können, dass der Bund eingreift, um die Gesundheitsversorgung in allen Bundesländern auf dem gleichen Niveau zu halten. Die Bundesländer haben direkte exekutive (Art. $30 \mathrm{GG}$ ) und eigene legislative Kompetenzen (Art. 70 Abs. 1 GG). Außerdem wirken die Länderregierungen über den Bundesrat an Gesetzen und Verordnungen des Bundes mit. Neben verfassungsändernden Gesetze bedürfen solche Gesetze, die Auswirkungen auf die Finanzen bzw. Organisations- und Verwaltungshoheit der Bundesländer haben, der ausdrücklichen Zustimmung des Bundesrats (Art. 84 Abs. 1 und Art. 104a. Abs. 4 GG). In der Gesundheitspolitik kann dies vor allem die Krankenhauspolitik und bestimmte Reformen des Ver- 
sicherungssystems betreffen - bspw. die aktuell diskutierte bundesweite Öffnung der AOKs. Den Bundesländern obliegt darüber hinaus der Sicherstellungsauftrag für die Krankenhausversorgung. In der deutschen Konsensdemokratie werden Entscheidungen unter Berücksichtigung mehrerer administrativer Akteure durch Verhandlung getroffen. In Bezug auf die vertikal (zwischen Bund und Bundesländern) und horizontal (zwischen Bundesländern) geteilte Entscheidungsmacht in Verhandlungen sprechen Scharpf et al. (1976) von Politikverflechtung. Diese führt dazu, dass Verteilungsfragen (also auch Finanzierungs- und Vergütungsstrukturen), die alle beteiligten Ebenen betreffen, lediglich Niveauverbesserungen und keine Strukturverbesserungen ermöglichen. Politikverflechtung bedeutet auch, dass administrative Strukturen (konkret also die Beteiligung von Bund, Ländern und Kommunen an der Gesundheitspolitik) dazu führen, dass Reformvorschläge nicht nur unter Berücksichtigung sachlicher Informationen entschieden werden, sondern auch unter dem Aspekt von Verteilungsfragen und ebenenspezifischen Interessen. Die Kritik an negativen Verteilungswirkungen hat gemeinsam mit konkreten Kompetenzinteressen der Bundesländer dazu geführt, dass die Politikverflechtung in der Krankenhausfinanzierung und -vergütung nach der Hochphase der 1970er Jahre - mit vereinzelten Ausnahmen - schrittweise reduziert wurde (Augurzky et al. 2018; Simon 2016). Nach Abschaffung einer Bundesmitverantwortung für Investitionen in Krankenhäuser 1984 folgte mit der Föderalismusreform I 2006 eine regulative Entflechtung. Ziel war es, die Anzahl an zustimmungspflichtigen Gesetzen zu senken. Dies hat es erleichtert, auch Krankenhausreformen nicht zustimmungspflichtig zu konstruieren (Bandelow und Hartmann 2014; Bandelow et al. 2019). Die Krankenhauspolitik ist aber weiterhin durch Planungs- und Finanzierungsfragen Teil des Mehrebenenkomplexes.

In Bezug auf die Krankenhausfinanzierung und -vergütung lassen sich darauf aufbauend zwei wesentliche Konfliktfragen in die- sem Mehrebenenspiel ausmachen, die auch die (Problematik der) Politikverflechtung illustrieren: Investitionsfinanzierung und Entgeltsystem. Die Investitionsfinanzierung sollte formal über Landesmittel bereitgestellt werden, wenngleich die Finanzierungssummen zurückgehen und die Länder wegen "Investitionsstau“ in Krankenhäusern und Verantwortungsdiffusion in der Bedarfsplanung und Sicherstellung häufig in der Kritik stehen (Leber und Scheller-Kreinsen 2018; Reiners 2018). Der Bund stellt aber punktuell auch Finanzmittel zur Verfügung, z. B. (aber nicht ausschließlich) über den Krankenhausstrukturfonds. Auch die Länder haben teilweise Sonderförderprogramme, um die Investitionen zu decken (Augurzky et al. 2018). Trotz der klaren formalen Trennung von Zuständigkeiten kommt es deshalb hier immer wieder zu gemeinschaftlicher Finanzierung von Krankenhäusern. Die Verflechtung führt an dieser Stelle dazu, dass Bundesländer auf externe Unterstützung hoffen. Diese kann darauf beruhen, dass der Bund die Finanzierung durch die Krankenkassen zulässt bzw. ermöglicht, dass Krankenhausträger aus den Betriebskosten Beiträge zu den Investitionen leisten können. Ähnliche Politikverflechtung gibt es bei Entgeltsystemen. Während die Bewertungsrelationen, also die geschätzten Behandlungskosten einer Falldiagnose, vom Bund festgelegt werden, sind die Landesbasisfallwerte das Ergebnis von Vereinbarungen auf Länderebene. Zusätzlich gibt es aber auch auf Bundesebene errechnete Bundesbasisfallwerte, die Korridore festlegen, innerhalb derer sich die Landesbasisfallwerte bewegen müssen. Beide (bzw. alle drei) Komponenten bestimmen die tatsächliche Vergütung, die ein Krankenhaus für einen Fall erhält. Auch hier kommt es in der Folge zu Verteilungsasymmetrien zwischen Ländern unter Einfluss des Bundes, da in Ländern mit niedrigeren Basisfallwerten Krankenhäuser auch weniger Geld für Leistungen bekommen. Der Bund übt durch die vorgegebenen Korridore Einfluss auf das generelle Niveau dieser Leistungsvergütung aus, ohne direkt auf die Verteilung zwischen den Ländern einzuwirken. 
Mit Blick auf die Kommunen kommt eine weitere föderale Konfliktlinie ins Spiel, die vor dem Hintergrund der Debatte um mehr Qualität der Krankenhausversorgung an Bedeutung gewinnt. Die Krankenhausinvestitionsfinanzierung wird von den Kommunen mitgetragen, die teilweise auch in ihrer Rolle als Krankenhausträger an der Krankenhauspolitik beteiligt sind. Allerdings existieren wiederkehrende Vorschläge, durch Schließungen kleinerer Krankenhäuser, Spezialisierung und Zentralisierung die ländliche Versorgung sicherzustellen. Die Kommunen stehen dieser Entwicklung - ebenso wie kommunale Parteivertretungen zumeist entgegen; hauptsächlich, weil die Bürger in ländlichen Versorgungsräumen Ängste um eine wohnortnahe Versorgung hegen. Dass mit einer Umstrukturierung der Krankenhauslandschaft aber auch ein Zuwachs an Qualität verbunden sein kann, ist diesen aufgrund der Emotionalität der Debatte nur schwer zu vermitteln. Folglich kommt es hier zu einer Mischung aus föderaler und parteipolitischer bzw. kommunalpolitischer Konfliktlinie und einer starken Salienz von regionaler Identität, die die Interessen der Bürger in einzelnen Regionen vertritt. Die Verflechtung der Zuständigkeiten von Kommunen, Bundesländern und Bund in der Krankenhausfinanzierung kann dabei zu einer Falle werden, die eine effiziente, qualitätsorientierte Krankenhauspolitik im gemeinsamen Sinne blockiert.

Die föderalen Strukturen führen also bei eigeninteressierten Akteuren regelmäßig zu suboptimalen Ergebnissen. Eine Lösung könnte in der vertrauensvollen Zusammenarbeit zwischen den beteiligten Akteuren liegen, um die jeweiligen Interessen angemessen in gemeinsamen Programmen zu berücksichtigen. Der deutsche Neokorporatismus ermöglicht eine solche Kooperation, indem die Akteure in Gremien und gruppenbildenden Organisationen regelmäßig zusammengebracht werden. Formalisierte wiederholte Gelegenheiten zur $\mathrm{Zu}$ sammenarbeit sind die Tagungen des in vielen Bundesländern bestehenden Krankenhausplanungsausschusses. Diesem gehören Vertreter von Kostenträgern, Ärztekammer, Landes- krankenhausgesellschaft und Krankenhausträgern an, die die Krankenhaus- und Investitionspläne gemeinsam ausarbeiten (Dreyer 2008). Wenngleich dieser Ausschuss formal Teil der Legislative ist, erfolgt die Abstimmung in enger Zusammenarbeit mit dem jeweiligen Ministerium, das die Letztentscheidung trägt. Als Gremium ist er deshalb zentral, indem er Zusammenarbeit und gegenseitiges Vertrauen ermöglicht, was im besten Fall auch dazu führt, dass sich eine gemeinsame Sicht auf Probleme und Lösungsstrategien entwickelt, die in einem langfristigen Gestaltungsprogramm - einer Vision - für die landesweite Krankenhausversorgung mündet.

\subsection{Interessengruppen und Selbstverwaltung}

Während die föderale Konfliktlinie in stärkerem Maß Krankenhausfinanzierung und in geringerem Maß Krankenhausvergütung fokussiert, sind Vergütungsströme und Fallpauschalen das hauptsächliche Konfliktthema der Akteure der Selbstverwaltung und jeweiligen Interessengruppen, darunter vor allem die Krankenhausgesellschaften, Krankenkassenverbände und Berufsverbände und -kammern. Hier gibt es einen großen Konsens in der Kritik am aktuellen DRG-System, wenngleich sich die Kritik aus unterschiedlichen Interessen speist. Ähnlich wie die DKG ist auch der GKV-Spitzenverband einerseits Akteur und andererseits Arena für gesundheitspolitische Entscheidungen, der mehrere, teilweise gegensätzliche Einzelinteressen in sich vereint und mit einer Stimme nach außen vertritt. Beide sind aber grundsätzlich Befürworter des DRG-Systems (anders als die Vertreter der Beschäftigten). Der GKV-Spitzenverband kritisiert am aktuellen DRG-System, dass Gelder oftmals nicht effizient und zweckgebunden verausgabt werden und sieht die Rückkehr zum Selbstkostendeckungsprinzip, wie sie bspw. in der Ausgliederung der Pflegekosten aus den Fallpauschalen vollzogen wird, als falschen 
Weg. Das DRG-System begrüßt der GKV-SV grundsätzlich als standardisiertes Finanzierungsinstrument, da es ihm ermöglicht, die zu finanzierenden Kosten zuverlässig abzuschätzen (GKV-Spitzenverband 2015).

Die Deutsche Krankenhausgesellschaft (DKG) hingegen bemängelt an der Berechnung der Fallpauschalen die unzureichende Berücksichtigung von Weiterbildungskosten, regionalen und strukturellen Unterschieden sowie tatsächlichen Krankenhausleistungen in der Behandlung von Patienten. Die mehrere Trägerschaften unter sich vereinende DKG vertritt damit das allen Krankenhausträgern gemeine Interesse, höhere Vergütungssummen für Krankenhausleistungen zu erhalten. Freigemeinnützige Träger wie bspw. die AWO formulieren Kritik vor allem im Hinblick auf die unzureichende Übernahme von Kosten für Personal durch die Kostenträger. Diese Kritik teilen auch die privaten Träger, die darüber hinaus im Gegensatz zu freigemeinnützigen und öffentlichen Trägern den wirtschaftlichen Erfolg des Krankenhauses in den Mittelpunkt stellen. Öffentliche Träger sind noch stärker um ihre Haushaltsdefizite besorgt, die sie im Zweifelsfall für Mehrausgaben in Krankenhäusern belasten müssen (AWO Bundesverband e. V. 2014; Bundesverband Deutscher Privatkliniken e. V. (BDPK) 2019; Deutsche Krankenhausgesellschaft (DKG) 2017; Eibelshäuser 2013).

Während Krankenhausträger (vor allem vertreten durch die DKG) und Kostenträger (vor allem vertreten durch den GKV-Spitzenverband) auf einer makropolitischen Ebene die Finanzierungs- und Vergütungsstrukturen diskutieren, geht es bei den Repräsentanten von Berufsgruppen, ihren Vereinigungen und Kammern um individuelle Auswirkungen von Vergütungsregelungen. Zusammenschlüsse von Berufsgruppen dienen der gemeinsamen Durchsetzung von Interessen. $\mathrm{Zu}$ den Erfolgselementen zählen Organisations- und Konfliktfähigkeit, also die Kapazität einerseits, eine bestimmte Interessensgruppe $\mathrm{zu}$ organisieren, was einfacher ist, je homogener und kleiner die Gruppe ist, da dann der Einzel- nutzen größer ist als bei größeren, diffusen Gruppen. Die Konfliktfähigkeit ist wiederum gleichzusetzen mit dem Drohpotenzial, das eine Gruppe nutzen kann, indem sie ihre Arbeit oder Aufgaben niederlegt (Klenk 2018). Die Zersplitterung der Gewerkschaften und Tarifgemeinschaft von ver.di und der „Spartengewerkschaft" des Marburger Bunds 2005 kann als ein Wendepunkt in der deutschen Interessensvertretung gesehen werden. Seitdem verhandelt der Marburger Bund teilweise begleitet von Ärztestreiks Tarifverträge im Krankenhausbereich - während nichtärztliches Personal im Krankenhaus keine Lobby hat. Der Marburger Bund setzt sich aber ähnlich wie die Bundesärztekammer und ver.di im Krankenhaus für eine bessere Berücksichtigung von Personalkosten im DRG-System ein. Sie kritisieren, dass höhere Aufwendungen für Leistungen am Patienten innerhalb einer DRG zulasten des Personals finanziert werden. Das DRGSystem habe somit nicht zur Kostensenkung beigetragen, sondern vielmehr eine Umverteilung von Ausgaben im Krankenhaus bewirkt, die zulasten von Personal und Patienten geht (Bundesärztekammer 2001; Marburger Bund 2018). Die Interessen der analysierten Akteure sind in - Abb. 16.1 anhand der identifizierten Konfliktlinien abgebildet. Neben den inhaltlichen Positionen verdeutlicht die Grafik, dass die Konfliktlinien keinesfalls trennscharf verlaufen, sondern dass sich die Akteure - wenn auch nicht mit primärer Aufmerksamkeit, aber doch häufig mit klarer Zuordnung - auf allen Ebenen bewegen.

Im Geflecht der bereits in $>$ Abschn. 16.2 beschriebenen föderalen Strukturen haben die Interessengruppen jeweils unterschiedliche Möglichkeiten der Einflussnahme. Während der Marburger Bund und ver.di über die Tarifverhandlungen Einfluss auf Vergütungspolitik nehmen können, sind die Träger über die jeweilige Landeskrankenhausgesellschaft ebenso wie die gesetzliche und private Krankenversicherung über ihre jeweiligen Verbände und die kommunalen Spitzenverbände im Krankenhausplanungsausschuss (oder Äquivalent) eines jeweiligen Landes vertreten, der sich im 


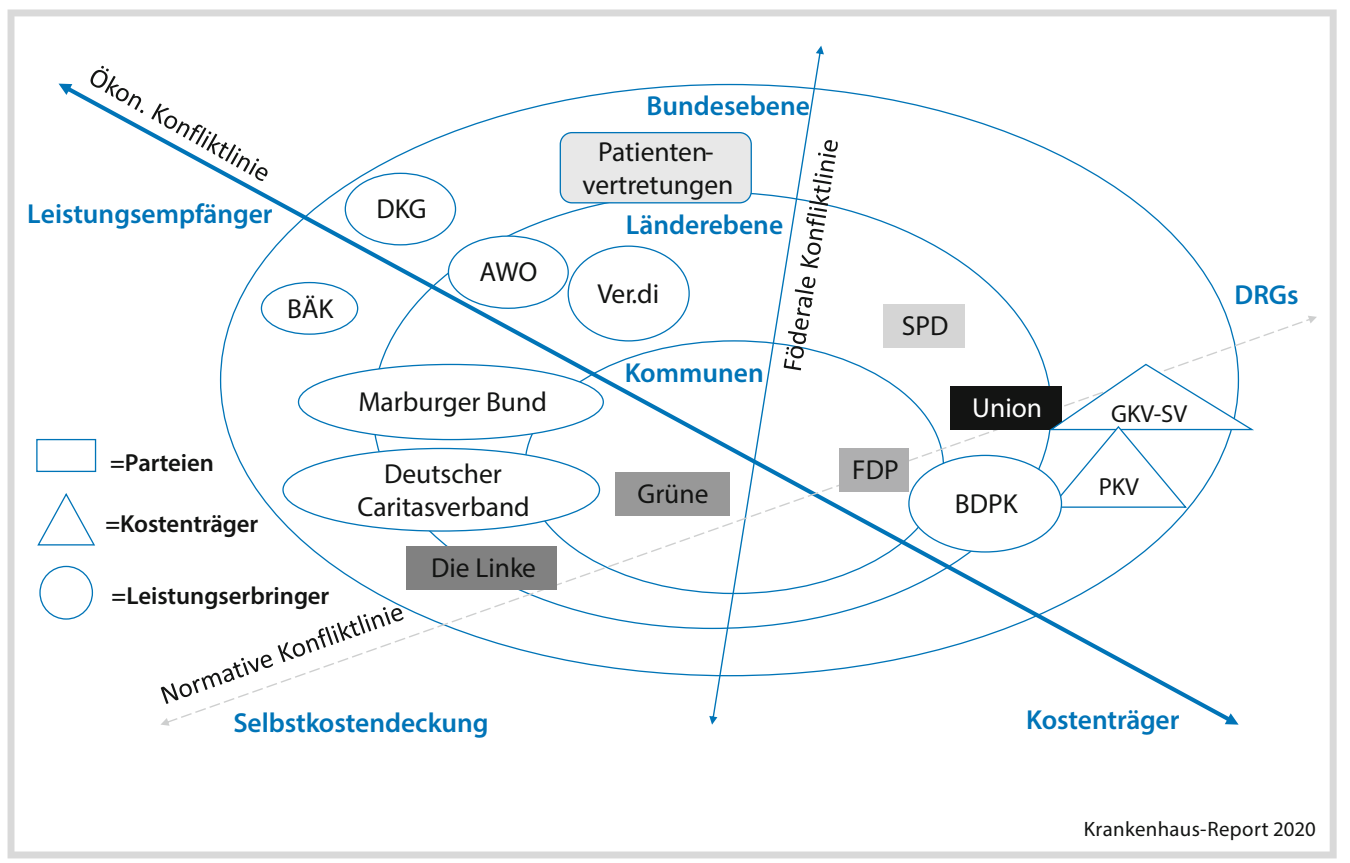

- Abb. 16.1 Positionen von Akteuren in der Krankenhausfinanzierungs- und -vergütungspolitik (Quelle: Vereinfachte Darstellung auf Basis eigener Auswertungen von Stellungnahmen, Wahlprogrammen, Positionspapieren)

Zuge der Erstellung des Krankenhausplans auch mit Krankenhausinvestitionen beschäftigt. In beiden Fällen zeigt sich der starke Korporatisierungsgrad, durch den Interessen institutionell in Entscheidungsprozesse eingebunden sind. Parteipolitische Akteure, die bereits in - Abb. 16.1 dargestellt sind und sich ursprünglich stärker an der normativen Konfliktlinie orientierten, spielen hier - zumindest in der aktuellen Debatte - nur eine begrenzte Rolle.

\subsection{Krankenhauspolitik in der Parteien-Arena}

Der Zeitraum zwischen ersten Diskussionen um die Einführung von Fallpauschalen und dem finalen Regelbetrieb mit diesem Vergütungssystem umfasste insgesamt etwa zwei Jahrzehnte und geschah unter Regierungskoalitionen von CDU/CSU und FDP (Kanz- ler Helmut Kohl bis 1998), SPD und Bündnis 90/Die Grünen (Kanzler Gerhard Schröder bis 2005) sowie der großen Koalition (CDU/ CSU und SPD) unter Kanzlerin Angela Merkel 2005 bis 2009. Auch die Regierungskoalitionen in einzelnen Bundesländern und damit verbunden die parteipolitischen Mehrheiten im Bundesrat unterlagen Veränderungen. Dies hatte zur Folge, dass ausgerechnet die aktuelle Koalition von Union und SPD bisher in keiner Phase über eine eigene Mehrheit im Bundesrat verfügte. Seit den 1960er Jahren hatten bisher alle Bundesregierungen zumindest zeitweise die Möglichkeit, zustimmungspflichtige Gesetze auch ohne Oppositionsparteien zu verabschieden. Die parteipolitischen Konflikte verlagern sich im Sinne der Politikverflechtung somit teilweise auf die föderale BundLänder-Ebene (vgl. > Abschn. 16.2). Gleichzeitig zeigt sich aber im beobachteten Zeitraum trotz wechselnder Mehrheiten eine Kontinuität in der Krankenhauspolitik in Bezug auf die Einführung von Fallpauschalen und die größere 
Vision für den Krankenhaussektor, was die Rolle von parteipolitischen Akteuren infrage stellt.

Analysiert man die Wahlprogramme einzelner Parteien, spiegelt sich die föderale Konfliktlinie darin, dass Krankenhauspolitik vor allem in Landtagswahlkämpfen und weniger in Bundestagswahlkämpfen ein Thema ist. Auffällig ist auch die über die Jahre hinweg zunehmende Aufmerksamkeit, die krankenhauspolitische Instrumente in den Wahlprogrammen zur Bundestagswahl erfahren und die vor allem seit der Einführung der Fallpauschalen zunimmt. Inhaltlich überraschend ist die hohe Homogenität der Forderungen. Tiefe Konflikte zwischen den Parteien sind in Finanzierungsund Vergütungsfragen kaum feststellbar; die über lange Zeit im Gesundheitswesen vorherrschende normative Konfliktlinie von Solidarität und Eigenverantwortung wurde mit dem Programm der programmatischen Gruppe überwunden. Einerseits lässt sich diese Beobachtung auf die Komplexität der Krankenhausfinanzierung zurückführen, die für Allgemeinpolitiker, die keine Experten für den Krankenhaussektor sind, schwer greifbar ist und dementsprechend keine starken Meinungen hervorruft. Andererseits erklärt sich dadurch auch die langfristige Zusammenarbeit innerhalb der programmatischen Gruppe über Parteigrenzen hinweg, die die Einführung der Fallpauschalen seit den 1990er Jahren vorantrieb. - Tab. 16.1 listet im Detail die Positionen der aktuell im Bundestag vertretenen Parteien in den letzten Jahren auf. Dabei ist zu beachten, dass eine nicht explizite Zustimmung zu einzelnen Instrumenten in der Krankenhausfinanzierung und -vergütung nicht mit einer Ablehnung dieser Instrumente einhergeht. Lediglich die expliziten Forderungen wurden aufgenommen. Dazu kommt, dass nur die Positionen zu monetären Aspekten der Krankenhauspolitik berücksichtigt wurden; Strukturreformen und die Ablehnung von Krankenhausprivatisierung bspw. wurden ob des Oberthemas dieses Beitrags vernachlässigt.

Koalitionsregierungen sind in der deutschen Konsensdemokratie in Bund und Ländern der Regelfall. Nicht umsonst werden Ko- alitionsverträge (KoaV) mittlerweile als wichtige und verlässliche Grundlage für die Gesetzgebung einer Legislaturperiode angesehen (Klüver und Bäck 2019). Bundespolitisch enthalten die KoaV seit 1998 u. a. aufgrund der starken Kompetenzbündelung auf Landes- und Kommunalebene nur wenige Verweise auf Krankenhauspolitik. Genannt werden das Ziel einer verbesserten Zusammenarbeit zwischen ambulantem und stationärem Sektor - konkret also zwischen Hausärzten, Fachärzten und Krankenhäusern (KoaV 2009, 2002, 1998), Vereinheitlichung der Vergütung für ambulante Leistungen in Krankenhäusern und bei niedergelassenen Ärzten (KoaV 2005), Evaluation der DRGs im Hinblick auf adäquate Berücksichtigung von Pflegeaufwand und Weiterbildung (KoaV 2009, 2005), die Ablehnung bundeseinheitlicher Preise und explizite Befürwortung von wettbewerblichen Strukturen (KoaV 2009), gesonderte Vergütung für Hochkostenfälle (KoaV 2013) und adäquate Nutzung der DRGs auch für Personalkosten bzw. Bereinigung der DRGs um Pflegepersonalkosten (KoaV 2018, 2013). Diese über lange Jahre unabhängig von Regierungen stabilen Ziele zeigen, dass die parteipolitische, normative Konfliktlinie bei der Krankenhausfinanzierung und -vergütung weit weniger wichtig ist als die föderale und ökonomische - und das, obwohl die Parteien in ihren Wahlprogrammen durchaus unterschiedliche Instrumente in der Krankenhausfinanzierung und -vergütung vertreten. Eine steigende Bedeutung dieser Dimension würde erst unter Regierungsbeteiligung weiterer Parteien mit stark divergierenden Interessen vorstellbar.

Darüber hinaus gibt es einige Parteien, die inhaltlich bestimmten Interessengruppen näherstehen als anderen (vgl. auch - Abschn. 16.3). Parteien dienen somit teilweise als Instrumentarium von privaten und staatsnahen Akteuren (etwa der Selbstverwaltung). Bspw. hat Die Linke eine große Nähe zu Gewerkschaften und setzt sich für einen bindenden Tarifvertrag für alle im Krankenhaus Beschäftigten ein. Während Die Linke sich für die Rückkehr zum Selbstkostendeckungs- 
- Tabelle 16.1 Parteipolitische Interessen in der Krankenhausfinanzierung und -vergütung (Quelle: Eigene Darstellung auf Basis ausgewählter Bundes- und Landtagswahlprogramme seit 2000, Auflistung der sechs aktuellen Bundestagsparteien in alphabetischer Reihenfolge)

\begin{tabular}{|c|c|c|c|c|c|c|c|}
\hline & AfD & $\begin{array}{l}\text { Bündnis 90/ } \\
\text { Die Grünen }\end{array}$ & CDU & CSU & Die Linke & FDP & SPD \\
\hline \multicolumn{8}{|l|}{ Investitionskosten } \\
\hline Gemeinsam von Bund und Ländern & ja & ja & ja & & ja & nein & ja \\
\hline Erhöhung von Investitionen & & ja & ja & ja & ja & ja & ja \\
\hline Pflichterfüllung durch Länder & ja & & & & & ja & \\
\hline Wettbewerb um staatliche Investitionsmittel & & & & & & ja & \\
\hline Investitionspauschale & & & $\begin{array}{l}\text { beides } \\
\text { erhalten }\end{array}$ & & ja & & \\
\hline Leistungsorientierte Einzelförderung & & ja & & & & & ja \\
\hline $\begin{array}{l}\text { Beteiligung der Krankenkassen (Teilmonistische } \\
\text { Finanzierung) }\end{array}$ & & ja & & & ja & & \\
\hline Monistische Finanzierung & & & & & & ja & \\
\hline Jährliche Sonderabgabe abschaffen & & & & & & ja & \\
\hline \multicolumn{8}{|l|}{ Entgeltsystem/DRG } \\
\hline Gegen DRG & ja & & & & ja & ja & \\
\hline Umstellung auf Rahmenplanung & & & & & & ja & \\
\hline $\begin{array}{l}\text { Berücksichtigung Preisentwicklung und Aus- } \\
\text { gleich Tarifsteigerung }\end{array}$ & ja & & ja & ja & & & \\
\hline Anwendung Tarifvertrag auf alle Beschäftigten & & & & & ja & & \\
\hline Gegen Budgetierung & & ja & & & & & \\
\hline Angleichung ambulant/stationär & & ja & & & & ja & ja \\
\hline Bedarfsgerechte Finanzierung & & & & & ja & & ja \\
\hline
\end{tabular}

prinzip einsetzt, will die FDP Fehlanreize verhindern, durch die Krankenhäuser Leistungen anbieten, die an anderen Stellen besser aufgehoben wären. Durch die eingeschränkte Rolle der Parteien im Rahmen von Koalitionsregierungen oder als Teil der Opposition vollzieht sich dieser Einfluss allerdings eher über die föderalen Wege von Bundesrat, Länderregierungen und Kommunen. Konkret spiegeln die Parteien auch die föderale Konfliktlinie wider, bspw. durch die Forderung nach gemeinsamer Finanzierungspolitik von Bund und Ländern. Erstaunlicher Konsens unter den Parteien besteht in weiten Teilen hinsichtlich der Finanzierungs- und Vergütungspolitik im Krankenhausbereich - von der Befürwortung erhöhter Investitionen über $\mathrm{Ab}$ lehnung von DRGs und Budgetierung bzw. hinsichtlich der Forderung nach bedarfsgerechter Finanzierung verbunden mit der Berücksichtigung der Preisentwicklung und dem Ausgleich von Tarifsteigerungen. 


\subsection{Ausblick}

Dieser Beitrag stellt die Komplexität der Konfliktlinien um Finanzierungs- und Vergütungsstrukturen im Krankenhaus sowie Einflussmöglichkeiten der einzelnen Akteure gegenüber. Die ökonomischen Interessen von Kostenträgern, Krankenhausträgern und Berufsverbänden sowie die politisch-normativen Ziele von parteipolitischen Akteuren werden in unterschiedlichem Maße auf Bundes-, Landesbzw. Kommunalebene verhandelt. Daraus ergibt sich abhängig von Blockade- oder Kooperationssituationen eine verminderte oder erhöhte Reformaktivität. Während die Einführung des Fallpauschalensystems eine strukturelle Veränderung darstellt, die über Jahrzehnte hinweg vorbereitet und schrittweise im Rahmen einer Vision von mehr Wettbewerb von einer programmatischen Gruppe implementiert wurde, bewegen sich aktuell diskutierte und verabschiedete Reformvorschläge auf einem inkrementellen Niveau. Konkret sind das etwa die Abkopplung der Pflegepersonalkosten von den Fallpauschalen und der Streit um die Prüfung von Krankenhausabrechnungen. Teilweise moniert wird auch die noch fehlende Anpassung der Investitionskostenfinanzierung an die wettbewerblichen Strukturen (Bohm und Schräder 2012). Allerdings gibt es im Moment keine klar dominierende, ressourcenstarke Gruppe mit zusammenhängendem Programm. Weitere Reformen in diese Richtung sind deshalb aktuell kaum durchsetzbar. Gleichzeitig sind die Strukturen aber noch existent und haben sich selbst verstetigt, trotz der Probleme, die diese Reformen mit sich brachten. So ist das von GKV, PKV und DKG gegründete Institut für das Entgeltsystem im Krankenhaus (InEK) als offizielles DRG-Institut mit der Definition und Pflege der Fallgruppen betraut. Eine Umstellung vom DRG-System auf ein alternatives Vergütungssystem würde dieses Institut obsolet machen oder müsste ihm eine neue Funktion zuweisen. Das Institut selbst steht hinter den Reformen, durch die es einst erschaffen wurde, und befürwortet das Fallpauschalensystem.
Die Verzahnung von ambulanter und stationärer Versorgung inklusive der Angleichung der Vergütung im ambulanten und stationären Sektor war ebenfalls Teil der Vision einflussreicher Akteure der letzten Jahrzehnte (Amelung und Janus 2005; Knieps 2005). Eine Angleichung der Vergütungsstrukturen gibt aber keine Antwort auf die strukturellen Probleme, die auch in der laufenden Debatte angesprochen werden. Darunter fällt die effiziente Aufteilung ambulanter und stationärer Behandlung, deren Problematik sich in einer Überbeanspruchung der Notaufnahme bei gleichzeitigen komplexen Behandlungen in separaten Facharztpraxen spiegelt. Die historisch gewachsenen Pfade sind nicht einfach $\mathrm{zu}$ durchbrechen und neue Pfade, die alte Probleme lösen, bedeuten auch immer neue Probleme, die verursacht werden. Eine komplette Umgestaltung des Finanzierungs- und Vergütungssystems - sofern dies überhaupt wünschenswert ist - kann nur dann gelingen, wenn die starken Eigeninteressen aller beteiligten Akteure aktiviert, in einem politischen Programm vereint und über eine soziale Gruppe verbunden werden, mit der sich diese Akteure identifizieren können. Die daraus entstehenden neuen Strukturen werden wiederum starke Selbsterhaltungseffekte erzeugen, ähnlich wie jene, die durch die Umstellung auf das Fallpauschalensystem entstanden sind.

Der kooperative Föderalismus bietet gleichermaßen Herausforderungen und Chancen für eine effektive Zusammenarbeit der in der Krankenhauspolitik relevanten Akteure. Eine Herausforderung ist die Gefahr von suboptimalen Ergebnissen durch Politikverflechtung, die darin besteht, dass bestimmte Vergütungs- und Finanzierungsinstrumente gemeinsam von Bund, Ländern und/ oder Kommunen gesteuert werden. Andererseits bieten gemeinsame Gremien, etwa der Krankenhausplanungsausschuss, die Möglichkeit konstruktiver Zusammenarbeit und der Bildung von Gruppen, die durch eine gemeinsame Sichtweise auf Probleme und Lösungen ein neues politisches Programm entwickeln können. Förderlich für eine innovative Ge- 
staltung in der Krankenhausfinanzierung und -vergütung sind zudem wissenschaftliche Erkenntnisse, bspw. aus der Gesundheitsökonomie oder Politikwissenschaft. Innerhalb dieser föderalen Strukturen versuchen die relevanten Akteure Einfluss auf Entscheidungen zu nehmen. Während Kostenträger, Krankenhausträger und Beschäftigte und ihre jeweiligen Vereinigungen und Organisationen hauptsächlich ökonomische Interessen vertreten (mehr Einnahmen für sich vs. weniger Einnahmen für die anderen Akteure), streben politische Akteure, darunter vor allem Parteien, nach elektoralem Erfolg und Karriereaufstieg. Parteien folgen dabei zumindest in der politischen Kommunikation normativen Vorstellungen von Gesundheits- und Krankenhauspolitik und beschränken sich dabei auf einfache, unkonkrete Aussagen, die öffentlichkeitswirksam und für Nichtexperten verständlich sind. Hier hat sich die die Konfliktlinien überlagernde Debatte um Qualität als dominant erwiesen. Reformvorschläge auch in Bezug auf Krankenhausfinanzierung und -vergütung werden folglich unter der Prämisse diskutiert, dass diese die Qualität im Krankenhaus steigern und verbessern sollten. Dieser Qualitätsdiskurs spricht darüber hinaus Patienten und ihre Vertretungen in entsprechenden politischen Entscheidungsgremien an, da ihr Interesse vor allem in dem Zugang zu qualitativ hochwertigen Gesundheitsleistungen liegt.

Damit verbunden bleibt die Herausforderung bestehen, Krankenhausfinanzierung und -vergütung in einer Weise zu organisieren, die die richtigen Anreize für eine qualitativ hochwertige Versorgung setzt, ohne die ökonomischen Interessen der einzelnen Akteure zu unterlaufen. Damit soll vor allem der Kritik begegnet werden, wonach Fallpauschalen unternehmerisches Verhalten vor leistungsorientiertem Bedarfsangebot begünstigen - und zwar unabhängig von politischen Systemen (Neby et al. 2015). An dieser Stelle schlägt dieser Beitrag drei mögliche Reformschritte vor. Der erste knüpft an die in den letzten Jahren aufkommende Debatte um evidenzbasierte Politik an, die Reformen auf Basis von wissen- schaftlichen, unabhängigen und objektiv erhaltenen Erkenntnissen propagiert. Im bestehenden DRG-System erfolgt dies bereits teilweise über die Erhebung der Leistungen und Kostendaten aller Krankenhäuser, die als eine Komponente in die Berechnung der DRGs einfließt. In Bezug auf qualitativ hochwertige Versorgung im Krankenhaus würde eine systematische Erforschung der Krankenhausorganisation unter Berücksichtigung qualitätsmessender Indikatoren aber eine Berechnung von Fallpauschalen ermöglichen, die von höherer Passgenauigkeit profitiert. Zu den Instrumenten der Messung von Qualität zählen auch patientenorientierte Studien sowie Zweitmeinungen von Ärzten, die bereits gesetzlich verankert sind. Um Alternativen zu Fallpauschalen $\mathrm{zu}$ erforschen, eignen sich außerdem Reallabore und Pilotkrankenhäuser, die unterschiedliche Finanzierungs- und Vergütungssysteme erproben und durch anschließende systematische Evaluation Evidenz für deren Effizienz und Qualität liefern können. In der Versorgungsplanung werden derartige Projekte bspw. vom RWI durchgeführt (Augurzky et al. 2019).

Ausgehend davon, dass in der Gesundheitspolitik häufiger von Verzahnung als von Trennung gesprochen wird, will dieser Beitrag für einen zweiten Reformvorschlag den Blick auf letzteres werfen: Im Fallpauschalensystem ergibt sich aus einem aktuellen Problem der diagnosebezogenen Vergütung, dass Entgelte unweigerlich an Diagnosen gekoppelt sind und die Krankenhäuser sowohl Diagnosen stellen als auch direkter Empfänger der Entgelte sind. Diese Doppelrolle begünstigt Diagnoseentscheidungen, die wirtschaftlich vorteilhaft für das Krankenhaus sind. Eine Trennung von Diagnose und Vergütung könnte diesen Anreiz umkehren.

Als Schritt in Richtung einer integrierten Versorgung von stationärem und ambulantem Sektor sind medizinische Versorgungszentren und ambulante, an Krankenhäuser angegliederte Praxen aktuell diskutierte Antworten. Dagegen wird über eine klarere Trennung von ambulanter und stationärer Versorgung zur effizienten Steuerung von 
Bedarfsströmen, die wir hier als dritten Reformvorschlag aufbringen wollen, nur vereinzelt gesprochen. Der Vorschlag, die ambulante Versorgung komplett auf den Hausarzt zu zentrieren und auf standardisierte Leistungen wie Impfungen und Erkältungsbekämpfung zu begrenzen sowie spezifische, nicht standardisierbare Leistungen auf Fachärzte im stationären Bereich (wenngleich dort auch ambulant behandelt wird) $\mathrm{zu}$ verlagern, steht dem dominanten Diskurs in Deutschland komplett entgegen, wenngleich es vereinzelte Forderungen danach gibt, die auch auf internationalen Vorbildern beruhen (Schmacke 2018; Schoen et al. 2004). Diese strukturelle Änderung müsste in das Fallpauschalensystem eingegliedert werden (Bandelow und Hornung 2019).

\section{Literatur}

Amelung VE, Janus K (2005) Modelle der integrierten Versorgung im Spannungsfeld zwischen Management und Politik. In: Klauber J, Robra BP, Schellschmidt H (Hrsg) Krankenhaus-Report 2005, Schwerpunkt: Wege zur Integration. Schattauer, Stuttgart, S 13-26

Augurzky B, Beivers A, Emde A, Halbe B, Pilny A, Straub N (2018) Stand und Weiterentwicklung der Investitionsförderung im Krankenhausbereich, Gutachten im Auftrag des Bundesministeriums für Gesundheit. RWI Leibniz-Institut für Wirtschaftsforschung, Essen. https://www.bundesgesundheitsministerium.de/ fileadmin/Dateien/5_Publikationen/Ministerium/ Berichte/Gutachten_Investitionsfoerderung Krankenhausbereich.pdf. Zugegriffen: 22. Juli 2019

Augurzky B, Beivers A, Breidenbach P, Haering A, Straub N (2019) Versorgungsplanung durch datenbasierte Marktraumanalysen am Beispiel von Notfallzentren. In: Klauber J, Geraedts M, Friedrich J, Wasem J (Hrsg) Krankenhaus-Report 2019, Schwerpunkt: Das digitale Krankenhaus. Springer, Berlin, Heidelberg, S 161174

AWO Bundesverband e.V. (2014) Stellungnahme des AWO Bundesverbandes e. V. zum Koalitionsvertrag zwischen CDU, CSU und SPD für die 18. Legislaturperiode des Deutschen Bundestages. https:// www.awo.org/sites/default/files/2017-01/AWO_ Stellungnahme_zum_Koalitionsvertrag.pdf. Zugegriffen: 15. Juli 2019

Bandelow NC, Eckert F, Rüsenberg R (2009) Qualitätsorientierung als "Megathema" der Zukunft? In: Bandelow NC, Eckert F, Rüsenberg R (Hrsg) Gesundheit 2030: Qualitätsorientierung im Fokus von Politik,
Wirtschaft, Selbstverwaltung und Wissenschaft. VS, Wiesbaden, S 13-26

Bandelow NC, Hartmann A (2014) Health policy prior to the German federal election of 2013: the party political marginalisation of a previously central topic in election campaigns. Ger Polit 23:371-385. https:// doi.org/10.1080/09644008.2014.953067

Bandelow NC, Hartmann A, Hornung J (2019) Winter is coming - but not yet. German health policy under the third Merkel chancellorship. Ger Polit 28:444-461. https://doi.org/10.1080/09644008. 2018.1512592

Bandelow NC, Hornung J (2019) Mehr Staat, weniger Selbstverwaltung, weniger Wettbewerb? Mut zur Evidenz - auch bei Governance-Fragen! Observer Gesundheit. https://observer-gesundheit. de/mehr-staat-weniger-selbstverwaltungweniger-wettbewerb-mut-zur-evidenz-auch-beigovernance-fragen/. Zugegriffen: 15. Juli 2019

Blum K (2018) Stationäre Versorgung. In: Thielscher C (Hrsg) Handbuch Medizinökonomie I: System der medizinischen Versorgung. Springer, Wiesbaden, S 1-19

Bohm S, Schräder WF (2012) Reformbedarf der regionalen Krankenhausplanung. G\&S Gesundheits- Sozialpolitik 66:16-24. https://doi.org/10.5771/16115821-2012-4-16

Bundesärztekammer (2001) Beschlussprotokoll des 104. Deutschen Ärztetages vom 22.-25. Mai 2001. https://www.bundesaerztekammer.de/aerztetag/ beschlussprotokolle-ab-1996/104-daet-2001/zupunkt-v-der-tagesordnung-taetigkeitsbericht-derbundesaerztekammer/drg-fallpauschalensystem/. Zugegriffen: 15. Juli 2019

Bundesverband Deutscher Privatkliniken e.V. (BDPK) (2019) Geschäftsbericht 2018/19. https://www. bdpk.de/media/file/3971.BDPK_Geschaeftsbericht_ 2018_2019.pdf. Zugegriffen: 15. Juli 2019

Deutsche Krankenhausgesellschaft (DKG) (2017) Patientenwohl und Daseinsvorsorge. Positionen der Deutschen Krankenhausgesellschaft (DKG) für die 19. Legislaturperiode des Deutschen Bundestags. https://www.dkgev.de/fileadmin/default/ Mediapool/1_DKG/1.6_Positionen/2017-03_DKGPositionspapier_19._Legislaturperiode.pdf. Zugegriffen: 15. Juli 2019

Dreyer M (2008) Die Länder müssen in der Verantwortung für die stationäre Versorgung bleiben. In: Klusen N, Meusch A (Hrsg) Zukunft der Krankenhausversorgung: Qualität, Wettbewerb und neue Steuerungsansätze im DRG-System, 1. Aufl. Nomos, Baden-Baden, S 71-80

Eibelshäuser M (2013) Vierundzwanzigster Zusammenfassender Bericht "Gesundheitswesen - Kliniken". http://starweb.hessen.de/cache/haushalt/24bericht-upkk.pdf. Zugegriffen: 15. Juli 2019 
GKV-Spitzenverband (2015) Stellungnahme des GKVSpitzenverbandes vom 20.08.2015 zum Entwurf eines Gesetzes zur Reform der Strukturen der Krankenhausversorgung (Krankenhausstrukturgesetz KHSG). https://www.bundestag.de/resource/blob/ 386080/0348ddcfe31811 b660017a01f8f7c66c/gkvspitzenverband-data.pdf. Zugegriffen: 15. Juli 2019

Heyder R, Malzahn J (2018) Krankenhauspolitik im neuen Koalitionsvertrag: Wohin geht die ordnungspolitische Reise? G\&S Gesundheits- Sozialpolitik 72:15-20. https://doi.org/10.5771/1611-5821-2018-2-15

Hornung J, Bandelow NC (2018) The programmatic elite in German health policy: collective action and sectoral history. Public Policy Adm Early View. https://doi. org/10.1177/0952076718798887

Klenk T (2018) Interessenlagen und Interessenpolitik im Gesundheitssektor. In: Spier T, Strünck C (Hrsg) Ärzteverbände und ihre Mitglieder: Zwischen Einflussund Mitgliederlogik. Springer, Wiesbaden, S 19-46

Klüver H, Bäck H (2019) Coalition agreements, issue attention, and cabinet governance. Comp Polit Stud. https://doi.org/10.1177/0010414019830726

Knieps F (2005) Perspektiven der Integrierten Versorgung in Deutschland - Der Ordnungsrahmen der GKV und die Aufgabe der Integration aus Sicht der Politik. In: Klauber J, Robra BP, Schellschmidt H (Hrsg) Krankenhaus-Report 2005, Schwerpunkt: Wege zur Integration. Schattauer, Stuttgart, S 27-36

Koalitionsvereinbarung (1998) Aufbruch und Erneuerung - Deutschlands Weg ins 21. Jahrhundert. Koalitionsvereinbarung zwischen der Sozialdemokratischen Partei Deutschlands und Bündnis90/Die Grünen. https://www.spd.de/fileadmin/Dokumente/ Beschluesse/Bundesparteitag/koalitionsvertrag_ bundesparteitag_bonn_1998.pdf. Zugegriffen: 15. Juli 2019

Koalitionsvertrag (2002) Erneuerung - Gerechtigkeit - Nachhaltigkeit. Für ein wirtschaftlich starkes, soziales und ökologisches Deutschland. Für eine lebendige Demokratie. Koalitionsvertrag zwischen SPD und Bündnis 90/Die Grünen 20022006. https://www.nachhaltigkeit.info/media/ 1248173898php7wc9Pc.pdf. Zugegriffen: 15. Juli 2019

Koalitionsvertrag (2005) Gemeinsam für Deutschland - mit Mut und Menschlichkeit. Koalitionsvertrag zwischen CDU, CSU und SPD. https://www.kmk. org/fileadmin/pdf/foederalismus/2005_11_11Koalitionsvertrag-Dok04.pdf. Zugegriffen: 15. Juli 2019

Koalitionsvertrag (2009) Wachstum. Bildung. Zusammenhalt: Der Koalitionsvertrag zwischen CDU, CSU und FDP. https://www.bmi.bund.de/SharedDocs/ Downloads/DE/Ministerium/koalitionsvertrag. pdf?_blob=publicationFile. Zugegriffen: 15. Juli 2019
Koalitionsvertrag (2013) Koalitionsvertrag zwischen CDU, CSU und SPD. https://www.bundesregierung. de/Content/DE/_Anlagen/2013/2013-12-17koalitionsvertrag.pdf?_blob=publicationFile. Zugegriffen: 15. Juli 2019

Koalitionsvertrag (2018) Ein neuer Aufbruch für Europa. Eine neue Dynamik für Deutschland. Ein neuer Zusammenhalt für unser Land. Koalitionsvertrag zwischen CDU, CSU und SPD. http:// www.handelsblatt.com/downloads/20936422/4/ koalitionsvertrag_final.pdf. Zugegriffen: 15. Juli 2019

Leber WD, Scheller-Kreinsen D (2018) Von der Landesplanung zur algorithmischen Marktregulierung. In: Klauber J, Geraedts M, Friedrich J, Wasem J (Hrsg) Krankenhaus-Report 2018, Schwerpunkt: Bedarf und Bedarfsgerechtigkeit. Schattauer, Stuttgart, S 101-130

Marburger Bund (2018) Marburger Bund fordert grundlegende Reform des DRG-Systems. https://www.marburger-bund.de/bundesverband/ pressemitteilung/marburger-bund-fordertgrundlegende-reform-des-drg-systems. Zugegriffen: 15. Juli 2019

Matusiewicz D (2019) Marketing im Gesundheitswesen - eine Einführung. In: Matusiewicz D, Stratmann F, Wimmer J (Hrsg) Marketing im Gesundheitswesen: Einführung-Bestandsaufnahme-Zukunftsperspektiven. Springer, Wiesbaden, S 3-24

Neby S, Lægreid P, Mattei P, Feiler T (2015) Bending the rules to play the game: accountability, DRG and waiting list scandals in Norway and Germany. EPA 1:127-148. https://doi.org/10.18278/epa.1.1.9

Paquet R (2019) Gesundheitspolitik der AfD - zusammengesuchtes Allerlei. Observer Gesundheit, Politische Analysen vom 15.03.2019. https:// observer-gesundheit.de/gesundheitspolitik-derafd-zusammengesuchtes-allerlei. Zugegriffen: 23. Juli 2019

Reiners H (2018) Bedarfsplanung und Sicherstellung - Dauerbaustelle der Gesundheitspolitik. G\&S Gesundheits- Sozialpolitik 72:21-25. https://doi. org/10.5771/1611-5821-2018-2-21

Scharpf FW, Reissert B, Schnabel F (1976) Politikverflechtung. Theorie und Empirie des kooperativen Föderalismus in der Bundesrepublik. Scriptor Verlag, Kronberg Berlin

Schmacke N (2018) Anforderungen an ein modernes Vergütungssystem aus medizinischer Sicht. G\&S Gesundheits-Sozialpolitik 72:29-31. https://doi.org/ 10.5771/1611-5821-2018-4-5-29

Schoen C, Osborn R, Huynh PT, Doty M, Davis K, Zapert K, Peugh J (2004) Primary care and health system performance: adults' experiences in five countries. Health Aff 23:W4-487-W4-503. https://doi.org/ $10.1377 /$ hlthaff.W4.487 
Simon M (2016) Die ökonomischen und strukturellen Veränderungen des Krankenhausbereichs seit den 1970er Jahren. In: Bode I, Vogd W (Hrsg) Mutatio- nen des Krankenhauses: Soziologische Diagnosen in organisations- und gesellschaftstheoretischer Perspektive. Springer, Wiesbaden, S 29-45

Open Access Dieses Kapitel wird unter der Creative Commons Namensnennung 4.0 International Lizenz (http:// creativecommons.org/licenses/by/4.0/deed.de) veröffentlicht, welche die Nutzung, Vervielfältigung, Bearbeitung, Verbreitung und Wiedergabe in jeglichem Medium und Format erlaubt, sofern Sie den/die ursprünglichen Autor(en) und die Quelle ordnungsgemäß nennen, einen Link zur Creative Commons Lizenz beifügen und angeben, ob Änderungen vorgenommen wurden.

Die in diesem Kapitel enthaltenen Bilder und sonstiges Drittmaterial unterliegen ebenfalls der genannten Creative Commons Lizenz, sofern sich aus der Abbildungslegende nichts anderes ergibt. Sofern das betreffende Material nicht unter der genannten Creative Commons Lizenz steht und die betreffende Handlung nicht nach gesetzlichen Vorschriften erlaubt ist, ist für die oben aufgeführten Weiterverwendungen des Materials die Einwilligung des jeweiligen Rechteinhabers einzuholen. 